\title{
ANALYTICAL MODELLING OF LINEAR SIZE EVOLUTION OF POWERFUL RADIO SOURCES
}

\author{
KRZYSZTOF T. CHYŻY \\ Astronomical Observatory of the Jagiellonian University \\ ul. Orla 171, 30-244 Kraków, Poland
}

We assume that two continuous, supersonic jets of plasma advance into a uniform surrounding medium of constant density and pressure. In terms of fluid dynamics their propagation is described by the kinetic model suitable for high Mach number flows, which likely occur in edge-brightened powerful radio sources. In this approximation the jet flowing energy is dominated by the bulk energy of thermal material. The end of the jet enters the undisturbed ambient medium and forms a front shock, where the supplied bulk energy is in situ transferred to relativistic particles and magnetic field.

In order to describe the time evolution of a forming cocoon we can divide the source age $T$ into consecutive time intervals $\Delta t$ that are small enough in comparison with the source age but longer than a characteristic time scale of microphysics processes. During that interval, a piece of a jet material reaches the jet head and shocks. Hydrodynamic simulation and analytical model by Hardee et al. (1992) show that the evolution of such a shocked material is closely approximated by sideways expansion only. Geometrically, the shock region can be approximated by an expanding cylinder.

Following Eilek \& Shore (1989) we assume that all kinds of energy in the shock region scale with the net input energy from the jet and that the factors of proportionality are constant during the whole radio source evolution considered. Taking into account the appropriate equations of state, we find the time evolution of all kinds of energy as well as the spectral luminosity of synchrotron emission in an expanding cylinder.

Finally we can evaluate the energy budget of the whole cocoon. A cocoon of the source of age $T$ consists of $n=T / \Delta t$ volume elements. The total energy of the cocoon is simply a sum of energy from all individual elements. By increasing the number of division $n$, the sum can be replaced by the integral over the time and evaluated. 
The model is applied to the cosmological evolution of linear sizes of powerful radio sources. Linear size $D$ of the model structure can be estimated as a product of the object's age and the longitudinal expansion in velocity of the cocoon. According to derived analytical formula the linear size of a radio source depends on the beam characteristics, the interaction of the beam with external medium, the source age $T$ and the cosmological epoch (through redshift $z$ ). An important conclusion about this relationship is a power dependence of linear size on spectral radio luminosity $P_{\nu}$ and redshift:

$$
D \propto P_{\nu}^{-0.27}(1+z)^{-2.1} .
$$

The model formula can be compared with relations estimated independently for a sample of 152 powerful radio galaxies and 173 extended quasars Chyży \& Zięba (1995). For observed radio galaxies, median linear sizes evolves with spectral radio power at $1.4 \mathrm{GHz}$ and redshift as:

$$
D \propto P_{1.4}^{-0.35 \pm 0.03}(1+z)^{-3.4 \pm 0.4} .
$$

The sample of quasars yields the relation:

$$
D \propto P_{1.4}^{-0.10 \pm 0.05}(1+z)^{-1.3 \pm 0.3} .
$$

In that case, the indices in the model dependence locate between those estimated for radio galaxies and quasars. The good correspondence of the model and actual sources confirms in fact the correctness of the model assumptions and approximations.

The decrease of linear sizes with redshift as described by (1) results from the increase of density of external gas in the earlier cosmological epochs. However, two effects contribute to this evolution. The first one arises from a higher in the past ram pressure, exerted on a source by external gas. Carefully examination of (1) reveals that the contribution of this effect is $73 \%$ of the total linear size-redshift dependence. The remainder $(27 \%)$ results from the redshift dependent radiation efficiency.

In the framework of the model, a slightly different relation of linear sizes for radio galaxies and quasars on redshift can be due to different cosmological evolution of the ambient gas density or longer duty cycle for radio galaxy central engines. Differences in luminosity relations can arise from shorter life time for more active objects.

Acknowledgments. This work was supported by the grant from Polish Committee for Scientific Research (KBN), grant no. PB/578/P03/95/09.

\section{References}

Chyży K.T., Zięba S. (1995) $A \& A, 303,420$

Eilek J.A., Shore S.N., 1989 ApJ 342, 187

Hardee P.E., White R.W., Norman M.L, Cooper M.A., Clarke D.A., 1992 ApJ, 387, 460 